\title{
Abuse of Cortisone in Leprosy
}

\author{
R. N. BASU \\ Medical Officer, Chilakallapalli Control Unit, \\ Srikakulam District, S. India
}

Cortisone the wonder drug when first used in 1950-55, has revolutionized the prognosis and line of treatment of several very serious diseases like acute rheumatoid and osteoarthritis, status asthmaticus, Addison's disease and a host of others and it has also found a prominent place in the treatment of certain stages of leprosy.

It is unfortunate that increasingly indescriminate use of cortisone in leprosy is leading to grave consequences. It has been almost a fashion to use cortisones for every sort of reaction in leprosy be it Tuberculoid, Borderline or Lepromatous, whether it is mild, moderate or severe. Some people even go so far as to use it at the first sign of intolerance to DDS for slight joint pain, dermatitis and any sort of eye complication resulting from the reaction and it is also unfortunate that those imperfectly trained join with the leprologists in excessive zeal for cortisone.

Summarizing the essential data about cortisone, it is one of the steroids of the adrenal cortex controlled by adeno-corticotrophic hormone secreted by the anterior pituitary. This water soluble crystalline sterol, or compound $\mathrm{E}$ of Kendall, has a structural formula of i 7 -hydroxy-I Idehydro-cortico-sterone and it plays an important part in stress reaction, affecting the metabolism of protein and controlling the metabolism of sodium and potassium. Cortisone has no direct effect in controlling the infection. HENCH in his Nobel Prize acceptance speech declared that cortisone is a therapy which shelters the patient and his tissues from the fire of infection but does quench the fire. So after using cortisone for a few days, there is a dramatic relief due to disappearance of the congestion of blood vessels, loss of the oedema and cellular exudation, and the patient thinks that he is cured but really the infection lurks inside.

Cortisone has definitely a place in the treatment of distressing Borderline and Tuberculoid reaction as well as in cases of erythema nodosum leprosum where it will bring prompt relief to the swelling of face, hands, and feet and to the involvement of bone and joints. But cortisone should not be used for routine non-lepromatous reaction where a course of potassium antimony tartrate is very effective in most of the cases. On the other hand prolonged use of cortisone in such cases will lead to depletion of potassium and retention of sodium, alteration of blood sugar level, altered protein excretion, etc., leading to irreversible processes. Moreover use of cortisone for treatment of slight reaction is irrational as it is a costly drug and it should be regarded as a very powerful armament to be used against a powerful enemy. If cortisone is used from the very beginning for treating simple ailments it will not yield the desired result at the opportune 
moment. Further, cortisone should not be used for treating progressive lepra reaction as it is more probably due to circulating tonins in blood upon which it has no effect.

Similarly it has been the fashion to use cortisone for simple dermatitis occuring after DDS therapy instead of calamine lotion with zinc which had hitherto given satisfactory results. It can be compared with driving off an old faithful servant to give place to a new smart one. We should not lose our sense of proportion and should use cortisone for cases of exfoliative dermatitis only.

Cortisone is prescribed at random for practically any eye complication resulting from reaction in leprosy. If it is used for conjunctivitis, the congestion clears like magic, but if there is any infection lurking inside, it will increase and there will be rebound phenomenon when the drops are withdrawn. It is also used in treatment for corncal ulcers, but actually it causes an ulcer to deteriorate. Superficial keratitis spreads deeper and decper with the use of cortisone.

In conclusion we may say that the steroids are tremendously powerful medicines which when judiciously and prudently applied in proper doses yield highly satisfactory results, but irrational and injudicious use of the steroids will lead to disaster. 